\title{
Dowling-Degos disease
}

INSERM

\section{Source}

INSERM. (1999). Orphanet: an online rare disease and orphan drug data base. Dowling-

Degos disease. ORPHA:79145

A rare, genetic, hyperpigmentation of the skin disease characterized by adulthood-onset of reticular, reddish-brown to dark-brown, macular and/or comedone-like, hyperkeratotic papules with hypopigmented macules, predominantly affecting flexural areas and, on occasion, progressing to involve trunk and acral regions. Histologically, epidermal acanthosis, thin, branch-like, rete ridges, and a tendency for acantholysis and pigmentary incontinence is observed. 